\section{TIVA in Cardiac Surgery}

\author{
Yatin Mehta ${ }^{1}$ Anil Karlekar ${ }^{2}$ Shweta Suri Kandpal ${ }^{1}$ \\ ${ }^{1}$ Medanta Institute of Critical Care and Anesthesiology, \\ Medanta The Medicity, Gurgaon, Haryana, India \\ 2Department of Cardiac Anaesthesia and Critical Care, \\ Fortis Escorts Heart Institute, New Delhi, India
}

J Card Crit Care:2021;5:5-6

Over the years, as for cardiac surgery, anesthesia too has undergone tremendous advancements in understanding of anesthetic agents mechanism and their monitoring. Studies have been underway with the ultimate goal of improving patient outcome, in terms of intraoperative hemodynamic parameters, myocardial protection, postoperative recovery, length of hospital stay, preservation of neurocognitive integrity, and long-term morbidity and mortality.

The cardioprotective effects of volatile anesthetics versus total intravenous anesthesia (TIVA) are controversial. Research has long been advocating volatile agents as preferred choice for myocardial protection. The beneficial effect of volatile anesthetics was termed "anesthetic preconditioning" (APC)

This occurred independent of changes in systemic and coronary hemodynamics, and persisted despite discontinuation of the volatile anesthetic before coronary artery occlusion, a "memory" period similar to that observed during ischemic preconditioning. Also, volatile anesthetics were shown to protect myocardium against ischemic injury when administered 24 to 72 hours before (termed "delayed" or "late" preconditioning) or immediately after (known as "postconditioning”) prolonged coronary artery occlusion. ${ }^{1}$

The Mortality in Cardiac Surgery Randomized Controlled Trial of Volatile Anesthetics (MYRIAD) trial, a randomized, single-blind trial, conducted at 36 centers in 13 countries did not reveal any significant difference in number of deaths at 30 days or at 1 year, in patients undergoing elective, isolated coronary artery bypass graft surgery, receiving intraoperative anesthesia with a volatile anesthetic or TIVA. ${ }^{2}$

Kapoor et al in a study comparing TIVA with desflurane in AVR showed no significant difference in troponin I (cTnI) and ischemia-modified albumin as a biomarker for myocardial injury, between both the study groups. However, the post-cardiopulmonary bypass cTnI level was significantly higher than baseline in the TIVA group,
Address for correspondence YatinMehta, MD, FRCA, Institute of Critical Care and Anesthesiology, Medanta The Medicity, Gurgaon, Haryana, India (e-mail: yatinmehta@hotmail.com).

demonstrating a cardioprotective ischemia preconditioning effect of desflurane. ${ }^{3}$

However, a Scandinavian registry of 10,535 patients undergoing a variety of cardiac surgical procedures revealed that patients with preoperative unstable angina and/or recent myocardial infarction, and thus already "preconditioned," did not show any difference in mortality between TIVA and volatile anesthetic groups. On the contrary, patients suffering from preoperative myocardial ischemia actually benefited from propofol anesthesia, due to its antioxidant effects. Cardiopulmonary bypass itself causes reperfusion injury that, when most severe, is clinically manifested as a systemic inflammatory response syndrome. The use of propofol during bypass is associated with a less adverse inflammatory profile than isoflurane, as shown by lower levels of cytokines and inflammatory biomarkers up to 24 hours post-surgery. ${ }^{4}$

In cardiac surgery, assessment of mitral regurgitation (MR) for valve replacement or repair is done under general anesthesia. A randomized controlled trial demonstrated that pre-existing MR might change differently from preoperative to intraoperative transesophageal echocardiogram according to the type of anesthetic agent. MR may be more underestimated with isoflurane anesthesia than with TIVA. ${ }^{5}$ Correct assessment of MR is important to take the decision whether to repair or replace the mitral valve and to assess the repair.

TIVA is highly effective in providing deep plane of anesthesia. Adequacy of TIVA depends on maintenance of brain concentrations in equilibrium with plasma levels. Al-Rifai suggested target-controlled infusions as the best way to achieve this state. They used the bolus/elimination/transfer principle to approximate a constant plasma level of drug. ${ }^{6}$

Also suggested was that clinicians quoted awareness as the reason to avoid TIVA; however, technical errors and poor application of knowledge were highlighted in the NAP5 published online

February 24, 2021
DOI https://doi.org/ $10.1055 / \mathrm{s}-0041-1723748$ ISSN 2457-0206. (c) 2021. Official Publication of The Simulation Society (TSS), accredited by International Society of Cardiovascular Ultrasound (ISCU).

This is an open access article published by Thieme under the terms of the Creative Commons Attribution-NonDerivative-NonCommercial-License, permitting copying and reproduction so long as the original work is given appropriate credit. Contents may not be used for commercial purposes, or adapted, remixed, transformed or built upon. (https://creativecommons.org/licenses/by-nc-nd/4.0/)

Thieme Medical and Scientific Publishers Pvt. Ltd., A-12, 2nd Floor, Sector 2, Noida-201301 UP, India 
report as the major cause of awareness during TIVA, and 75\% of these cases would have been prevented by suitable education and training. National Institute of Health and Clinical Excellence (NICE)has recommended deployment of a processed electroencephalography device when administering TIVA and NAP5 emphasized that this is particularly necessary in patients who require neuromuscular paralysis. However, prevention of excessive hypnosis is probably the most beneficial outcome of using such devices during TIVA. ${ }^{6}$

Hannam et al compared the hemodynamic profiles of etomidate and propofol for the induction of anesthesia in cardiac surgery and concluded that etomidate provides superior hemodynamic stability to propofol. ${ }^{7}$ However, a reduction of $50 \%$ of the infused volume using the $2 \%$ formulation is possible and may be preferable for the maintenance of anesthesia in patients in whom a larger lipid load might be considered undesirable. ${ }^{8}$

Interindividual variability in pharmacodynamic response represents a more challenging aspect of using TIVA. Adequate training of the clinician is essential and close clinical monitoring of the patient remains an important part of the anesthetist's role. With wide popularity of minimally invasive/robotic cardiac surgery along with fast tracking in cardiac surgery, the role of TIVA is going to become even more significant.

\section{Conflict of Interest}

None.

\section{References}

1 Pagel PS. Myocardial protection by volatile anesthetics in patients undergoing cardiac surgery: a critical review of the laboratory and clinical evidence. J Cardiothorac Vasc Anesth 2013;27(5):972-982

2 Landoni G, Lomivorotov VV, Nigro Neto C, et al; MYRIAD Study Group. Volatile anesthetics versus total intravenous anesthesia for cardiac surgery. N Engl J Med 2019;380(13):1214-1225

3 Kapoor PM, Taneja S, Kiran U, Rajashekhar P. Comparison of the effects of inhalational anesthesia with desflurane and total intravenous anesthesia on cardiac biomarkers after aortic valve replacement. Ann Card Anaesth 2015;18(4):502-509

4 Irwin MG, Chung CKE, Ip KY, Wiles MD. Influence of propofolbased total intravenous anaesthesia on peri-operative outcome measures: a narrative review. Anaesthesia 2020;75(Suppl 1): e90-e100

5 Ahn JH, Ahn HJ, Yi JW. Total intravenous anesthesia maintained the degree of pre-existing mitral regurgitation better than isoflurane anesthesia in cardiac surgery: a randomized controlled trial. J Clin Med 2019;8(8):1104

6 Al-Rifai Z. Principles of total intravenous anaesthesia: practical aspects of using total intravenous anaesthesia. BJA Educ 2016;16(8):276-280

7 Hannam JA, Mitchell SJ, Cumin D, et al. Haemodynamic profiles of etomidate vs propofol for induction of anaesthesia: a randomised controlled trial in patients undergoing cardiac surgery. Br J Anaesth 2019;122(2):198-205

8 Dewandre J, Van Bos R, Van Hemelrijck J, Van Aken H. A comparison of the $2 \%$ and $1 \%$ formulations of propofol during anaesthesia for craniotomy. Anaesthesia 1994;49(1):8-12 